\title{
Documentos electrónicos y normalización: Información y conocimiento. Perspectivas de futuro
}

José Vicente Rodríguez Muñoz

Departamento de Información y Documentación

Universidad de Murcia

«Lo que se concibe bien se enuncia claramente y las palabras para decirlo llegan con claridad»

(N. Boileau, Art poétique)

\subsection{Resumen}

Las Tecnologías de la Información están forjando nuevas formas de comunicación entre las personas, están generando cambios en la forma en la que se estructura la Sociedad; en el modo de trabajar, el lugar en que vivimos, como nos divertimos, la educación de nuestros hijos, etc. En esa Sociedad, la clave del éxito estará en la facilidad de acceso al conocimiento.

Una cuestión transcendente es el establecimiento de normas, de metodologías y de formas de hacer que sean ampliamente aceptadas y asumidas por todos para alcanzar un adecuado grado de igualdad en el acceso al conocimiento.

Aunque las perspectivas son halaguieñas, también tenemos que preguntarnos de que manera la tecnología será integrada en nuestras vidas. Las pruebas que habrá que pasar serán, seguramente, no sólo pruebas de inventiva, sino también de carácter. (Autor)

Palabras clave: Conocimiento. Infoespacio. Infopistas. Información. Normas. Redes.

\subsection{Abstract}

The Information Technologies are forging new forms of communication between the persons, they are generating changes in the way that Society is structured; in the manner of working, the place in which we lived, as we amused ourselves, the education of our children, etc. In that Society, the key of the success will be in the access facility to the knowledge. 
An issue transcendence is the procedures establishment, of methodologies and in the ways of causing that will be widely accepted and assumed all over to reach an adequate degree of equality in the access knowledge.

Though the perspectives are flattering, also must ask us of the fact that way the technology will be integrated in our lives. The tests that there will be that to pass will be, certainly, not only inventiveness tests, but also of character. (Author)

Keywords: Infospace. Infoways. Information. Knowledge. Network. Standard.

\section{Introducción}

No parece ocioso recordar, si acaso para entrar en materia, que lo que se viene en llamar las Nuevas Tecnologías, o Tecnologías de la Información o, a veces, simplemente Informática, hoy en día totalmente unidas a las telecomunicaciones en lo que denominamos Telemática, no son unas meras prótesis, unas meras herramientas más en el devenir histórico del ser humano, sino que realmente están cambiando nuestra manera de pensar, nuestra manera de comunicarnos, en definitiva nuestra manera de expresar el conocimiento.

No es causal, por tanto, que hayan sido las causantes ya de una revolución social, pues han dado lugar al paso de la economía de la información, sobre la anterior economía industrial, dentro de lo que se denomina en la actualidad, Sociedad de la Información.

Pues bien, cuando todavía estamos tratando de definir a esta latente Sociedad de la Información, ya se habla de una nueva revolución que nos llevará a la Sociedad del Conocimiento, el problema es que, como ya hemos señalado, todavía estamos definiendo los caminos de la entrante sociedad de la información, estamos en procesos de reestructuración en las organizaciones, en definitiva en continuos procesos de cambio, por tanto aún no disponemos de todas las respuestas ni sabemos exactamente a donde iremos, o mejor dicho a donde llegaremos.

La realidad es irrefutable, cada día nos vamos adentrando en una gran red de información de alcance mundial, o lo que es lo mismo de un sistema de comunicaciones que sobrepasa las fronteras tanto geográficas como políticas de los países, cuestión esta que puede, de algún modo, estar modificando los acontecimientos mundiales. Así, mientras que tecnologías como el teléfono o la televisión unen de una forma que hasta hace tan siquiera una generación no se hubiera imaginado, las nuevas redes de transmisión de datos, como Internet, no son otra cosa que adelantos de lo que queda por venir. 
Aun por complejas que nos parezcan las actuales redes de comunicación, no dejan de ser los componentes embrionarios de lo que se está preparando. La construcción de redes, las cuales se vienen denominando autopistas de la información o infopistas, se viene desarrollando dentro de cada país o región, pero el objeto final será el de una red universal, un mundo virtual del conocimiento, configurado a través de la información soportada en el entramado neuronal de ordenadores y personas.

Hoy en día estamos pues, presenciando la creación de un servicio fundamentalmente nuevo y que se está universalizando, al igual que lo hizo el suministro de electricidad que permitió, de este modo, el desarrollo de la era industrial. Este no es sino un servicio público de información, que está dando lugar a lo que ya muchos autores denominan economía del conocimiento, en definitiva, como ya hemos señalado, entramos en la era del conocimiento.

Este servicio cuando esté plenamente universalizado, permitirá que consumidores y proveedores estén enchufados en un circuito tal, que los primeros extraerán la información necesitada y los segundos recibirán en forma de una factura periódica el abono por sus servicios.

Se ve, de forma clara, que este será un sector amplio y estratégico, y las expectativas que crea están dando lugar a verdaderas contiendas entre las empresas que quieren dominar el mercado de la información y el de las comunicaciones, el cual según las previsiones puede ascender en unos años a varios miles de billones de pesetas.

Parece claro que esta, ya cercana, era del conocimiento afectará de manera profunda nuestra vida, pero hoy todavía no sabemos claramente cómo. Modificará el modo como trabajamos, el lugar en que vivimos, como nos divertimos, la educación de nuestros hijos, ... Pero lo que es más importante, en una sociedad donde el acceso al conocimiento será la llave del poder y la riqueza, también determinará quiénes prosperarán y quiénes no.

Ahora bien, decir que este adelanto no es más que una red de comunicaciones basada en un par de tecnologías, o llamarle autopista o carretera de la información, nos lleva a no entender la cuestión en toda su extensión. Como ya mucha gente expresa, estamos hablando de un nuevo mundo, de una forma de vivir, estamos hablando del infoespacio, un universo electrónico con una plétora de información, o lo que es mejor aún pleno de conocimiento.

Por ello cabe señalar y cuestionar las consecuencias de las normas y los estándares, o planteado de otra forma la ausencia de ellos, cuestión esta clave para la entrada del infoespacio, ya que esa falta de normativa es la que ha originado un retraso en la incorporación masiva de las tecnologías de la información 
y su desarrollo en el campo de la gestión de información y, consecuentemente, en la documentación, lo que, entre otras cosas, genera desconfianza.

Pues bien, una forma de actuar sin normas, lo que podríamos definir como optimización del tratamiento individual, o el enfoque caso a caso, ha dado lugar a la aparición de determinados elementos disfuncionales, o asimetrías, entre las que se podría destacar el elevado grado de incompatibilidad de los sistemas informáticos, con los inconvenientes que ello representa desde los puntos de vista que a continuación podemos describir:

- Dependencia de constructor, con lo que implica una situación de monopolio de oferta cuando se aborda la ampliación de un equipo de partida.

- Dificultad en la transportabilidad de las aplicaciones, que conduce a que distintos grupos de desarrollo de proyectos están trabajando en múltiples unidades departamentales en la realización de aplicaciones iguales o muy semejantes, con el consiguiente despilfarro de recursos que la mayoría de las veces son escasos

- Menor rentabilidad de las inversiones en formación, que son aprovechadas plenamente sólo si el trabajador que la ha recibido para un determinado entorno permanece fiel al mismo a lo largo de su vida en la organización. Lo cual es muy improbable.

- El bajo nivel de comunicación e integración entre centros por razones asociadas a la diversidad de arquitecturas implicadas y del modus operandi. Este inconveniente tiene importantes consecuencias en la gestión interna de las organizaciones, y de cara al exterior, en tanto que tienen que conectarse con el.

En definitiva, si nos detenemos a contemplar las aplicaciones de las Tecnologías de la Información, podemos decir que éstas se orientan principalmente a la mejora de la gestión administrativa y de los sistemas de información en general, en el sentido de una mayor productividad: automatización de procedimientos que implican el manejo de elevados volúmenes en los que se ha llegado a una situación problemática, cuya resolución no puede demorarse más.

De ahí que digamos que las Tecnologías de la Información constituyan un recurso, y en buen número de casos, el último recurso para aliviar situaciones de paralización que se presentan en el curso de las operaciones en los sistemas administrativos y de información en general. Por el contrario, no han aparecido, sino hasta hace poco tiempo, aplicaciones dirigidas a innovar o modernizar la propia gestión, esto es, la incorporación de herramientas informáticas dirigidas al apoyo al rendimiento con los objetivos de un plan estratégico global (no específicamente de Tecnologías de la Información). 


\section{Funciones de la normalización}

Todo lo expuesto nos hace reflexionar sobre la necesidad, sino de llevar la normalización hasta extremos de ahogo normativo, pues no debemos de caer en las tentaciones de detentar el poder, e imponer, si, al menos, programar estrategias, recomendaciones, dictámenes, informes, estándares, etc.

Se trata pues de dar paso a planteamientos más proactivos, que sean reflejo de la idea de que el objetivo de incorporación de las Tecnologías de la Información puede encontrar un firme basamento en una adecuada estrategia de uso de las mismas. De esta manera podemos decir que la utilización de las Tecnologías de la Información debe orientarse además de a la mejora de la gestión administrativa, hacia los siguientes aspectos:

- Mejora de la planificación y la toma de decisiones.

- Mejora de la prestación de los servicios en relación con los usuarios.

- Abrir el camino de la desconcentración de las estructuras organizativas.

En este sentido, tanto el Estado Español, como la Unión Europea a través de comisiones y organismos creados a tal efecto, están actuando con la orientación anteriormente mencionada, esto es, orientativa. De manera genérica las funciones encomendadas a estas comisiones las podemos reunir en las siguientes:

a) Identificar áreas prioritarias de aplicaciones de los sistemas y tecnologías de la información proponiéndolas como tales a los distintos estamentos.

b) Proponer a los órganos competentes de los distintos estamentos las medidas oportunas para la creación de bases de datos de interés.

c) Definir y proponer normas de intercambio de información y de adquisición, para su adopción por los diversos estamentos.

d) Emitir informes o dictámenes que soliciten los estamentos relativos a sus estrategias o proyectos de carácter tecnológico.

e) Informar, a petición de los utilizadores, en materia de contratación de bienes y servicios informáticos y tecnológicos.

f) Organizar seminarios, conferencias y otras actividades orientadas a la mejora de la formación de los empleados y usuarios.

h) Desarrollar las actuaciones de coordinación, o de colaboración entre los estamentos, precisas para el cumplimiento de los objetivos señalados.

i) Proponer a los órganos competentes la realización de estudios de viabilidad y proyectos piloto de servicios telemáticos.

j) Coordinar el desarrollo, realización e implantación de las redes telemáticas.

Scire. $3: 1$ (ene. -jun. 1997) 139-151 
k) Colaborar en la identificación, desarrollo e implantación de proyectos de modernización de los servicios que impliquen el uso de sistemas, equipos y servicios telemáticos, cuando sea requerido para ello por los órganos competentes.

1) Crear y mantener actualizado un fondo documental relativo a las materias del ámbito de actuación de los estamentos.

m) Identificar las necesidades en materia de infraestructuras, servicios y recursos humanos asociadas a la consecución de los fines de los estamentos y proponer a los órganos competentes medidas para su satisfacción, dentro de un marco conceptual común.

\section{Modelo para el desarrollo de normativa}

A la vista de lo dicho, se pone de manifiesto que los órganos de elaboración y desarrollo de la política tecnológica deben tener el carácter de consultivos, a los que les corresponde básicamente funciones de estudio, informe y propuesta. Aunque en algunos casos se puedan producir informes que deban ser preceptivos, como puede ocurrir si de elaborar anteproyectos de Ley se trata, en el caso de la Administración Pública.

En este sentido cabe destacar el desarrollo de normativa, y no poco relevante, en cuanto a la utilización de técnicas electrónicas, informáticas y telemáticas por parte de la Administración General de Estado Español, a través del Real Decreto 263/1996, que desarrolla la Ley 30/92, de 26 de noviembre, de Régimen Jurídico de las Administraciones Públicas y del Procedimiento Administrativo Común, la cual contiene una decidida apuesta por la abierta incorporación de las técnicas anteriormente mencionadas en la actividad administrativa y, en especial, a las relaciones entre los ciudadanos y las Administraciones Públicas. De este Real Decreto merece destacar los Artículos 3 y 4 del Capítulo I y todo el Capítulo II.

Si tratáramos de abstraer un modelo para desarrollar un cierto esquema de modus operandi en cuanto a llevar a cabo un proceso de incorporación de las Tecnologías de la Información y consecuentemente de modernización, podríamos plantear las siguientes etapas o fases como una adecuada representación de la realidad:

$1^{\text {a }}$ Etapa: Identificación de un área de interés general o línea estratégica en la que se considera apropiado realizar una actuación común.

2 Etapa: Desarrollar, adaptar o adoptar una Metodología para abordar la problemática asociada al área identificada, incluyendo su aplicación en un proyecto piloto. 


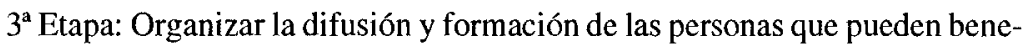
ficiarse de la formación en la Metodología.

$4^{a}$ Etapa: Proporcionar herramientas automatizadas para el aprendizaje y el uso de la Metodología, bien mediante la personalización de otras preexistentes o por desarrollo ad-hoc.

$5^{\text {a }}$ Etapa: Establecer, en su caso, un esquema de homologación de las herramientas anteriores proporcionadas por el mercado.

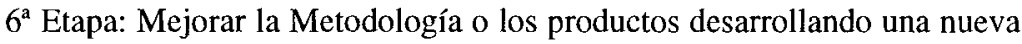
versión y repetir el ciclo anterior, a partir de la fase correspondiente.

\section{Perspectivas de futuro}

Es evidente que las cuestiones que afectan a los Sistemas de Información y Comunicación están adquiriendo progresivamente un alcance europeo e, incluso, mundial. Ello se pone de manifiesto en documentos de tanto calado como el Libro Blanco de Delors sobre "Crecimiento, Competitividad, y Empleo. Retos y pistas para entrar en el Siglo XXI" o el posterior informe Bangemann sobre "Europa y la Sociedad Global de la Información".

En particular, este último propone el desarrollo de diez aplicaciones para lanzar la Sociedad de la Información, esto es, diez líneas estratégicas. Se trata de las siguientes:

$1^{\circ}$ ) El teletrabajo: mayor empleo y nuevos trabajos para una sociedad móvil.

$\left.2^{\circ}\right)$ Educación a distancia: Educación permanente para una sociedad en mutación.

$3^{\circ}$ Una red de Universidades y Centros de Investigación: La interconexión del potencial de pensamiento e investigación europeo.

$4^{\circ}$ ) Servicio telemáticas para las PYMES: Un instrumento potente para relanzar el crecimiento y el empleo en Europa.

$5^{\circ}$ ) Gestión del tráfico por carretera: Carreteras electrónicas para una mayor calidad de vida.

$6^{\circ}$ ) Control del tráfico aéreo: Vías aéreas electrónicas para Europa.

$\left.7^{\circ}\right)$ Redes de Asistencia Sanitaria: Sistemas de asistencia sanitaria más baratos y más efectivos para los ciudadanos europeos.

$8^{\circ}$ ) Licitación electrónica: Una administración más eficaz y económica.

$9^{\circ}$ ) Red transeuropea de Administraciones públicas: Una administración mejor y más barata. 
$\left.10^{\circ}\right)$ Autopistas urbanas de la información: La sociedad de la Información en casa.

Con estas líneas estratégicas podemos analizar el futuro, lo que hemos denominado infoespacio desde cinco enfoques ligeramente diferentes de la forma en que se debe configurar la tecnología a fin de poder ofrecer el conocimiento de un modo eficaz, en esa esperada Sociedad de la Información.

La visión del consumidor particular. Desde el punto de vista del consumidor, el infoespacio aparece como vehículo de servicios informáticos públicos con el que se puede obtener educación y diversión a petición, tener acceso a los servicios del gobierno, comprar desde casa, etc.

La visión del grupo de trabajo. Si se forma parte de una organización, se verá el infoespacio como un medio de colaboración por ordenador, como una ayuda para los muchos trabajadores que colaboran a través del tiempo y del espacio en un subconjunto de su trabajo.

La visión de la organización. Desde la organización, desde la globalidad de la organización, la perspectiva del infoespacio es la de la automatización de los procesos. Este debe permitir automatizar los procesos organizacionales con la eliminación de las jerarquías, para todos los trabajadores que intervienen en ellos.

La visión de la gestión del conocimiento. Desde el punto de vista de un trabajador que desee tener acceso al conocimiento, el infoespacio debe representar la totalidad del proceso de recogida, agrupación y distribución de diversas formas de conocimiento: formación, casos, documentos técnicos, normas y reglamentos, incluso el viejo y buen sentido común.

La visión del trabajador intelectual (visto a éste como un consumidor de conocimiento con el objeto de generar valor añadido). Desde el punto de vista de este trabajador que está en su puesto de trabajo, el infoespacio es como los sistemas de apoyo al rendimiento. Y lo que es más importante, estos conectan al trabajador con los otros componentes o visiones del infoespacio. De este modo el trabajador podrá conectarse con los consumidores que están interactuando con los servicios informáticos públicos, con colegas que desean colaborar mediante el ordenador, con la totalidad del proceso organizacional a través de la automatización y con cualquier tipo de conocimiento al que una persona desee acceder.

Cabría preguntarse, ¿pero, por qué ahora?. Partes del infoespacio han ido saliendo a la luz estos últimos años, presentándose en forma de visiones creativas o académicas. Ya en el año 1945, Vannevar Bush director de la Oficina de Investigación Científica y Desarrollo de EE.UU., escribió un artículo en el que imaginaba un ordenador de sobremesa desde el que se podía acceder instantáneamente a todo el conocimiento almacenado en el mundo entero. Esta posibilidad no fue considerada por nadie como un adelanto palpable. Tres años más tarde, el 
lado más tenebroso de esta posibilidad tecnológica fue incorporado en la novela de George Orwell, 1984: un gobierno que utilizaba una especie de «infoespacio» para vigilar y dominar a las personas.

Entonces, ¿qué hay de diferente ahora? Para empezar, gran parte de la resistencia a la tecnología, resistencia por razones de cultura, de edad o de costumbre, se ha reducido con el cambio de generación. Además, ahora las personas de todas las edades se han acostumbrado a tener un acceso instantáneo a la información y a las noticias; en el mercado actual las normas sobre servicio al cliente han mejorado hasta tal punto, que los consumidores se muestran sorprendidos cuando las empresas no pueden responder rápidamente a sus exigencias.

Desde un punto de vista de los medios técnicos, la relación precio/rendimiento ha mejorado enormemente en la pasada década, y ahora estamos presenciando inversiones gigantescas por parte de los proveedores de infraestructura con objeto de poner en marcha estos mercados. En EE.UU., la Administración que subió al poder en 1993 cuenta con un vicepresidente versado en las redes informáticas de alta velocidad.

Todos esos adelantos juntos significan que ya han entrado de lleno en escena tres protagonistas del infoespacio: Los proveedores de contenidos, las empresas de infraestructura y los consumidores de información.

- Proveedores de contenidos: Los principales protagonistas están ahora prestos a crear y adaptar contenidos con destino al infoespacio: educación, televisión, películas, publicaciones, vídeo juegos, conversaciones, etc.

- Empresas de infraestructura: Las posibilidades tecnológicas de la infraestructura o canal de distribución se han multiplicado por mil en la pasada década. Ya se está hablando de canales de 3 gigabits de capacidad, es decir, 3.000 millones de bits por segundo. ¿Qué quiere decir eso?, pues por ejemplo; poder transmitir cada segundo 100 exploraciones tridimensionales de rayos $\mathrm{X}$ para otros tantos pacientes de cáncer, o de transmitir 1.000 fotografías tomadas por satélite a los investigadores. Traducido esto a páginas escritas a máquina, supondría 100.000 páginas por segundo: lo que quiere decir, que toda una enciclopedia podría ser transmitida en un abrir y cerrar de ojos. En resumen, el propio infoespacio está posibilitado por una capacidad informática masiva, barata y de amplitud de banda.

- Consumidores de información. Cada vez es mayor el interés de los consumidores por las redes de servicio interactivas. Por ejemplo, el tráfico en Internet y otros servicios de información en línea ha crecido muchísimo. Internet ha pasado de los 9.800 abonados que tenía en 1986 y sólo en EE.UU., a las varias decenas de millones de abonados de todas las nacio

Scire. 3 : 1 (ene. -jun. 1997) 139-151 
nes en la actualidad. Actualmente se transmiten más de una decena de billones de bytes al mes.

Por otro lado, la reducción de las limitaciones tradicionales del consumo se ha realizado gradualmente, y el sorprendente ritmo de crecimiento de los servicios de red muestra que los consumidores y los trabajadores están aprovechando muy bien todas las posibilidades. Naturalmente, el hombre sólo puede conocer a través de los sentidos, y el infoespacio parte del supuesto de que el consumo sólo se puede virtualizar por medio de los sentidos visual y auditivo. El consumo de esos elementos viene limitado, o venía limitado, por el tiempo y el espacio. Es decir, a una persona que vivía en España le resultaba muy difícil acudir a un concierto en Berlín; un estudiante de Tokio no podía fácilmente acudir a una conferencia en Londres. También han existido limitaciones de forma. Por ejemplo, para consultar una enciclopedia hacía falta que alguna persona o institución poseyera físicamente esa serie de volúmenes.

Las limitaciones del consumo auditivo empezaron a ser eliminadas hace muchos años gracias a la radio y al teléfono, debido a que se trataba de señales de poca amplitud de banda: para transmitir la voz sólo se requiere una capacidad de 55 bits por segundo. En cambio, las señales visuales tienen relativamente una gran amplitud de banda: la foto fija, incluso de baja resolución, requiere un millón de bits por segundo; las imágenes en movimiento requieren 30 millones de bits por segundo. Pero en el infoespacio esas limitaciones serán suavizadas, si no eliminadas por completo, cuando gran parte de nuestra vida visual vaya siendo «virtualizada»: películas, actuaciones teatrales, conferencias, obras de arte, ilustraciones, libros, mapas, etc. Todo esto puede ser conseguido en parte gracias al cable de fibra óptica que puede contener 25.000 canales, cada uno de los cuales tiene la posibilidad de transmitir hasta 1.000 millones de bits por segundo.

Pero, ¿cuánto tiempo hará falta para que el infoespacio se haga realidad? Se puede intentar responder a esa pregunta calculando la cantidad de tiempo que los servicios públicos actuales tardaron en penetrar en el hogar. En general, los servicios que necesitan una amplia infraestructura terrestre han ido avanzando con una relativa lentitud. Por ejemplo, la electricidad tardó más de 70 años en llegar al 50\% de los hogares. Al teléfono le hicieron falta 50 años para entrar en el $70 \%$ de los hogares; a la televisión por cable le costó 40 años superar el nivel del $60 \%$. Por contra, los servicios que no necesitan una importante infraestructura terrestre suelen penetrar mucho más rápidamente. La radio, en 10 años, penetró en el $70 \%$ de los hogares; la televisión alcanzó ese nivel en 15 años. Debido a que en los medios de distribución del infoespacio hay una gran diversidad de componentes, algunas de sus partes alcanzarán la madurez antes que otras. 
Podemos suponer que el infoespacio va a seguir una curva de implantación similar a la de los otros servicios públicos, pero eso no quiere decir que vayamos a tener que esperar mucho tiempo. Los futuros historiadores escribirán que la curva de implantación del infoespacio empezó a principios de los años cincuenta, con las primeras aplicaciones comerciales del ordenador. En la década de los noventa el infoespacio alcanzará probablemente el mismo nivel que tenía la electricidad en los años veinte, el teléfono en los cuarenta y la televisión en los cincuenta; es decir, estará listo para penetrar en la mayoría de los hogares en unos pocos años, tras una larga y lenta fase de incubación. Parece que al final, las empresas van a empezar a recoger realmente las ganancias por el dinero invertido en equipos informáticos.

Es seguro que la mayoría de las empresas y sectores se verán finalmente afectados por el infoespacio, y que muchas industrias y puestos de trabajo serán desplazados de un modo permanente e incluso eliminados. Como mínimo, el 75\% de los sectores de actividad presentan cierto potencial para las nuevas alternativas «virtuales» de banda ancha. No es necesario señalar que las actividades físicas, extracción de materiales, transformación y transporte, no son virtualizables. Sin embargo, las empresas que se dedican a vender, presentar, transformar o agregar información, es muy probable que dentro de relativamente muy pocos años realicen parte de su actividad dentro del infoespacio. No es ninguna exageración afirmar que muchas personas deberán pasarse el resto de su vida, empezando desde ahora, adaptándose continuamente a lo que el infoespacio está forjando.

Las sociedades suelen seguir un ciclo de creación, destrucción y reconstrucción. El hombre dedica años e incluso siglos a crear instituciones, estructuras políticas y sociales, entidades económicas, naciones, grupos étnicos, clases sociales, artes, valores, etc.; luego, todo se desintegra hecho pedazos y se vuelven a unir las piezas de una nueva manera.

Cuando se ha levantado la nueva estructura, podemos reconocer algunas partes de ella, pero la estructura es irreconocible. Al cabo de cierto tiempo, no recordamos ya el aspecto que tenía la anterior.

La nueva estructura está construida sólo en parte. Hoy en día no sabemos discernir qué piezas pertenecen a la antigua estructura y cuáles forman parte de la nueva. Pero sí parece cierto que se está imponiendo la noción de que el reconocimiento es el capital del futuro.

A resultas de esto cambiará todo nuestro mundo. Pero, ¿hacia dónde se dirige todo esto?, y sobre todo, ¿cuáles son las consecuencias de los acontecimientos que se están produciendo a nuestro alrededor y sobre los cuales la mayoría de la gente sabe que tiene muy poco control?

Scire. $3: 1$ (ene. -jun. 1997) 139-151 
Sólo una visión general, sólo una materialidad sistemática, puede proporcionarnos poder sobre el sistema. Hoy en día todos de algún modo participamos en la creación de nuevas estructuras y nuevos sistemas; y, si no los entendemos y nos anticipamos a ellos, nos convertimos en sus víctimas.

Cuando empezaron a imponerse los ordenadores en los años sesenta, muchos analistas temieron que la automatización llevase a más trabajadores al desempleo. En aquella época, los defensores de la automatización afirmaban que ocurriría lo contrario: que la automatización llevaría a la creación de nuevos puestos de trabajo.

Viéndolo desde la perspectiva de hoy en día, podemos comprobar que la automatización llevó realmente a la gente al desempleo, empujándola hacia nuevos sectores de actividad. Este es un hecho que se dió ya anteriormente. Por ejemplo, en la agricultura, la aplicación de la automatización hizo que los labradores dejasen el campo y se trasladasen a las fábricas. A su vez, la automatización de las fábricas hizo que los obreros se pasasen a la banca y a otros empleos del sector de los servicios. Hoy en día, el extendido sector de los servicios emplea a más del $70 \%$ de la población trabajadora.

La automatización seguramente hará que esa gente tenga que pasar a algún otro sector, pero ¿a cuál? Al llegar al cambio de siglo, el hombre habrá ya reorganizado los tres principales sectores de la economía: agricultura, industria, servicios. Entonces, ¿ $¿$ dónde ir?, evidentemente no hay una respuesta unívoca y simple a esta pregunta, tal vez, esta esté, en el trabajo que día a día tenemos todos por delante.

Pero, lo que se sabe de seguro es que, en esta nueva estructura los trabajadores gozarán de una gran ventaja. Aquellos que estén más "enterados" pueden tener confianza en la victoria.

Pero ¿qué pasará con los perdedores? Podemos estar seguros que, si se deja a la sociedad actual desarrollarse según su propia naturaleza, abocará a una desigualdad económica aún mayor, incluso entre las naciones. Este es el momento de preguntarnos qué es lo que vamos a hacer, por qué y, como se puede lograr que los adelantos económicos y tecnológicos enriquezcan nuestra vida en lugar de empobrecerla.

$\mathrm{Al}$ ir desarrollando tecnologías cada vez más potentes y centrar nuestra atención sobre la forma en que esas tecnologías se integran en la vida de la organización y en el medio en que vivimos, también tenemos que preguntarnos sobre la forma en que esa tecnología ha de ser integrada en la vida de las personas: de esas personas que constituyen nuestras organizaciones y nuestras comunidades sociales, electrónicamente definidas y geográficamente dispersas. Las pruebas que se tendrán que pasar no serán sólo pruebas de inventiva, sino también de carácter. 


\section{Referencias}

Ang, J. ; Pavri, F. (1994). A Survey and Critique of the Impacts of Information Technology. // International Journal of Information Management. (1994).

Comisión Europea (1994). Crecimiento, Competitividad, Empleo. Retos y pistas para entrar en el siglo XIX. Libro Blanco. CBruselas : ECA-CE-CEEA, 1994.

Gates, Bill (1995). Camino al futuro // Bill Gates ; Myhrvold N. ; Rinearson P. (cols); Francisco Ortiz Chaparro (trad). Madrid : McGraw-Hill, Interamericana de España, 1995.

Guide for managing electronic records from an archival perspective (1996). International Council on Archives, Committee on Electronic Records, 1996.

Linares, Julio (1995). Autopistas inteligentes // Julio Linares, Francisco Ortiz Chaparro. Madrid : Fundesco, 1995.

MAP (1995). Metodología de planificación y desarrollo de sistemas de información. MÉTRICA v. 2.1. Tecnos : Madrid, 1995.

Scire. 3 : I (ene. -jun. 1997) 139-151 